\title{
SOME NEW COMPLETE SETS OF IDENTITIES FOR AFFINE AND METRIC SPACES
}

\author{
BY JACK LEVINE
}

1. Introduction. Complete sets of identities have been obtained for the components of the affine and metric normal tensors and also for the components of the affine, metric, and projective curvature tensors. In addition to these identities, a complete set is known for the components of the first covariant derivative of the affine curvature tensor. ${ }^{*}$

In this paper a complete set of identities is obtained for the components of the second covariant derivative of the affine curvature tensor, and also a complete set for the first and second covariant derivatives of the metric curvature tensor.

2. The First Covariant Derivative. We shall now obtain a complete set of identities for the components $\dagger B_{i j k l, m}$ of the first covariant derivative of the metric curvature tensor with components $B_{i j k l}$. In terms of the $g_{i j}$ and their derivatives, we have

$$
\begin{aligned}
B_{i j k l}=\frac{1}{2} & \left(\frac{\partial^{2} g_{i k}}{\partial x^{j} \partial x^{l}}-\frac{\partial^{2} g_{i l}}{\partial x^{j} \partial x^{k}}-\frac{\partial^{2} g_{j k}}{\partial x^{i} \partial x^{l}}+\frac{\partial^{2} g_{j l}}{\partial x^{i} \partial x^{k}}\right) \\
& +g_{a b}\left(\Gamma_{i k}^{a} \Gamma_{j l}^{b}-\Gamma_{i l}^{a} \Gamma_{j k}^{b}\right) .
\end{aligned}
$$

By expressing (1) in metric normal coordinates, differentiating and evaluating at the origin, we obtain

$$
B_{i j k l, m}=\left(g_{i k, j l m}+g_{j l, i k m}-g_{i l, j k m}-g_{j k, i l m}\right) / 2 .
$$

The quantities $g_{i j, k l m}$ are the components of the third extension of the $g_{i j}$. The following identities form a complete set for these components:

$$
\begin{gathered}
g_{i j, k l m}=g_{i i, k l m}=g_{i j, p q r}, \\
g_{i j, k l m}+g_{i k, j l m}+g_{i l, j k m}+g_{i m, j k l}=0,
\end{gathered}
$$

* T. Y. Thomas, Differential Invariants of Generalized Spaces, Cambridge University Press, 1934, pp. 81, 114, 132, 138.

$\dagger$ In this section and the next small Latin indices have the range $1,2, \cdots, n$. 
where $p q r$ is any permutation of $\mathrm{klm}$. From (2) and (3), it is not difficult to show that

$$
\begin{aligned}
& B_{i j k l, m}=-B_{j i k l, m}=-B_{i j l k, m}, \\
& B_{i j k l, m}+B_{i j l m, k}+B_{i j m k, l}=0, \\
& B_{i j k l, m}+B_{i k l j, m}+B_{i l j k, m}=0 .
\end{aligned}
$$

The equation (2) can be solved for the $g_{i j, k l m}$, the solutions being

$$
\begin{aligned}
6 g_{i j, k l m}= & B_{i k j l, m}+B_{i k j m, l}+B_{i l j m, k}+B_{j k i l, m} \\
& +B_{j k i m, l}+B_{j l i m, k} .
\end{aligned}
$$

In obtaining (5) we use the identities (3) and also

$$
g_{k l, i j m}=g_{i j, k l m}+g_{j m, k l i}+g_{m i, k l j},
$$

this latter identity being a consequence of (3). Starting with (5) and (4), it is possible to obtain (2) and (3), and hence we have the following theorem.

THEOREM 1. The identities (4) constitute a complete set of identities for the components $B_{i j k l, m}$ of the covariant form of the first covariant derivative of the curvature tensor of a metric space.

3. The Second Covariant Derivative. We now consider the complete sets of identities for the components of the second covariant derivatives of the affine and metric curvature tensors. The affine case is first discussed. In terms of the components of the affine normal tensors, we have

$$
B_{j k l, m, p}^{i}=A_{j k l m p}^{i}-A_{j l k m p}^{i}+\psi_{j k l m p}^{i},
$$

where

$$
\begin{aligned}
\psi_{j k l m p}^{i}= & A_{a l m}^{i} A_{j k p}^{a}+A_{a l p}^{i} A_{j k m}^{a}+A_{a m p}^{i} A_{j k l}^{a}+A_{a l k}^{i} A_{j m p}^{a} \\
& +A_{j l a}^{i} A_{k m p}^{a}+A_{a j k}^{i} A_{l m p}^{a}-\left(A_{a k p}^{i} A_{j l m}^{a}+A_{a k m}^{i} A_{j l p}^{a}\right. \\
& \left.+A_{a m p}^{i} A_{j l k}^{a}+A_{a k l}^{i} A_{j m p}^{a}+A_{j a l}^{i} A_{k m p}^{a}+A_{j k a}^{i} A_{l m p}^{a}\right) .
\end{aligned}
$$

A complete set of identities for the components $A_{j k l m p}^{i}$ is given by the formulas

(9) $\quad A_{j k l m p}^{i}=A_{k j l m p}^{i}=A_{j k r s t}^{i}$, (rst is any permutation of $\left.l m p\right)$.

$$
\begin{aligned}
A_{j k l m p}^{i}+ & A_{j l k m p}^{i}+A_{j m l k p}^{i}+A_{j p l m k}^{i}+A_{k l m p j}^{i}+A_{k m l p j}^{i} \\
& +A_{k p m l j}^{i}+A_{l m p j k}^{i}+A_{l p m j k}^{i}+A_{m p j k l}^{i}=0 .
\end{aligned}
$$


By differentiating covariantly the components $B_{j k l, m}^{i}$ in a complete set of identities which these components satisfy, we obtain

$$
\begin{gathered}
B_{j k l, m, p}^{i}=-B_{j l k, m, p}^{i}, \quad B_{j k l, m, p}^{i}+B_{k l j, m, p}^{i}+B_{l j k, m, p}^{i}=0 \\
B_{j k l, m, p}^{i}+B_{j l m, k, p}^{i}+B_{j m k, l, p}^{i}=0
\end{gathered}
$$

If in (7) the indices $m$ and $p$ be interchanged and the resulting equation be subtracted from (7), there results

$$
\begin{aligned}
B_{j k l, m, p}^{i}-B_{j k l, p, m}^{i}= & B_{j k l}^{a} B_{a m p}^{i}-B_{a k l}^{i} B_{j m p}^{a} \\
& -B_{j a l}^{i} B_{k m p}^{a}-B_{j k a}^{i} B_{l m p}^{a} .
\end{aligned}
$$

This latter identity, (12), is one of Ricci's identities. Use is made of the relations

$$
B_{j k l}^{i}=A_{j k l}^{i}-A_{j l k}^{i}
$$

in obtaining it. The $A_{j k l m p}^{i}$ can be obtained in terms of the $B^{\prime}$ s by use of (7), (9), (10), the result being

$$
\begin{aligned}
10 A_{j k l m p}^{i}= & 2 \lambda_{j k l m p}^{i}+\lambda_{j k m l p}^{i}+\lambda_{j k p l m}^{i}+\lambda_{k j l m p}^{i}+\lambda_{k j m l p}^{i} \\
& +\lambda_{k j p m l}^{i}+\lambda_{l j m k p}^{i}+\lambda_{l j p m k}^{i}+\lambda_{j k l p m}^{i} \\
& +\lambda_{m j p k l}^{i}+\lambda_{j k m p l}^{i},
\end{aligned}
$$

where we have put

$$
\lambda_{j k l m p}^{i}=B_{j k l, m, p}^{i}-\psi_{j k l m p}^{i} .
$$

In (15) the $\psi$ are to be thought of as being expressed in terms of the $B_{b c d}^{a}$, this being done with the aid of the formula

$$
A_{b c d}^{a}=\left(B_{b c d}^{a}+B_{c b d}^{a}\right) / 3 .
$$

The components $B_{b c d}^{a}$ satisfy the following identities which form a complete set:

$$
B_{b c d}^{a}=-B_{b d c}^{a}, \quad B_{b c d}^{a}+B_{c d b}^{a}+B_{d b c}^{a}=0 .
$$

From (11), (12), (17), and (14) it can be shown after rather lengthy calculations that the complete set of identities (9), (10) is satisfied, and also the equations (7) can be obtained. Hence we have the following theorem.

Theorem 2. The identities (11), (12), (17) constitute a complete set of identities for the components $B_{j k l, m, p}^{i}$ and $B_{j k l}^{i}$. 
The identities for the components $B_{i j k l, m, p}$ of the second covariant derivative of the metric curvature tensor with components $B_{i j k l}$ will now be considered. In terms of the components of the metric normal tensors and the $g_{i j}$ and their extensions it is found that

$$
B_{i j k l, m, p}=g_{i a}\left(A_{j k l m p}^{a}-A_{j l k m p}^{a}\right)+h_{i j k l m p},
$$

where

$$
\begin{aligned}
h_{i j k l m p}= & g_{i a}\left(A_{j k p}^{b} A_{b l m}^{a}+A_{b l p}^{a} A_{j k m}^{b}-A_{j l p}^{b} A_{b k m}^{a}\right. \\
& \left.-A_{b k p}^{a} A_{j l m}^{b}+B_{j k l}^{b} A_{b m p}^{a}\right) \\
& -B_{i b k l} A_{j m p}^{b}-B_{i j b l} B_{k m p}^{b}-B_{i j k b} B_{l m \dot{p}}^{b} .
\end{aligned}
$$

Also

$$
B_{a b c d}=g_{a c, b d}-g_{b c, a d},
$$

and $A_{b c d}^{a}$ can be obtained in terms of the $g$ 's by means of (16) and (20). We find that

$$
\begin{aligned}
& g_{d k}\left(A_{i j l m p}^{k}-A_{i l j m p}^{k}\right) \\
& \quad=(1 / 2)\left(g_{d j, i l m p}+g_{i l, d j m p}-g_{d l, i j m p}-g_{i j, d l m p}\right)+a_{d i j l m p},
\end{aligned}
$$

where

$$
\begin{aligned}
a_{d i j l m p}= & A_{i l m g_{b d, j p}}^{b}-A_{i j m}^{b} g_{b d, l p}+A_{i l p g_{b d, j m}}^{b} \\
& -A_{i j p}^{b} g_{b d, l m}-R_{i j l g_{b d, m p}}^{b},
\end{aligned}
$$

and $g_{i j, k l m p}$ are the components of the fourth extension of the $g_{i j}$. If we place

$$
C_{i j k l m p}=a_{i j k l m p}+h_{i j k l m p},
$$

then (18) can be rewritten, by means of (21),

$$
\begin{aligned}
B_{i j k l, m, p}= & (1 / 2)\left(g_{i k, j l m p}+g_{j l, i k m p}-g_{i l, j k m p}-g_{j k, i l m p}\right) \\
& +C_{i j k l m p} .
\end{aligned}
$$

It is evident that $C_{i j k l m p}$ can be expressed in terms of the $g$ 's. The $g_{i j, k l m p}$ satisfy the complete set of identities

$$
\begin{aligned}
& g_{i j, k l m p}=g_{j i, k l m p}=g_{i j, q r s t},(q r s t \text { is any permutation of } k l m p), \\
& g_{i j, k l m p}+g_{i k, j l m p}+g_{i l, j k m p}+g_{i m, j k l p}+g_{i p, j k l m}=0 .
\end{aligned}
$$


From (4) and (12) we can obtain the identities

$$
\begin{aligned}
& B_{i j k l, m, p}=-B_{j i k l, m, p}=-B_{i j l k, m, p}, \\
& B_{i j k l, m, p}+B_{i k l j, m, p}+B_{i l j k, m, p}=0 \\
\text { (26) } \quad B_{i j k l, m, p}+B_{i j l m, k, p}+B_{i j m k, l, p} & =0 \\
B_{i j k l, m, p}-B_{i j k l, p, m}=B_{i a m p} B_{j k l}^{a} & -B_{i a k l} B_{j m p}^{a} \\
& -B_{i j a l} B_{k m p}^{a}-B_{i j k a} B_{l m p}^{a} .
\end{aligned}
$$

It is found that we can solve for the components $g_{i j, k l m p}$ by the use of (24) and (25), obtaining

$$
\begin{aligned}
10 g_{i j, k l m p}= & E_{i k j l m p}+E_{j k i l m p}+E_{i k j m l p}+E_{j k i m l p} \\
& +E_{i k j p m l}+E_{j k i p m l}+E_{i l j m k p}+E_{j l i m k p} \\
& +E_{i l j p m k}+E_{j l i p m k}+E_{i m j p k l}+E_{j m i p k l}
\end{aligned}
$$

and

$$
E_{i j k l m p}=B_{i j k l, m, p}-C_{i j k l m p} .
$$

The following identities are useful in obtaining (27)

$$
\begin{gathered}
g_{m p, i j k l}=g_{i j, k l m p}+g_{i k, j l m p}+g_{i l, j k m p}+g_{j k, i l m p} \\
+g_{j l, i k m p}+g_{k l, i j m p},
\end{gathered}
$$

these being derivable from (25). The quantities $E$ are expressed in terms of the $g_{i j}, B_{i j k l}, B_{i j k l, m, p}$. Now we can obtain (25) and (24) by means of (27) and (26), and as the $B_{i j k l}$ have the complete set of identities

$$
B_{i j k l}=-B_{j i k l}=-B_{i j l k} ; B_{i j k l}+B_{i k l j}+B_{i l j k}=0,
$$

we have the following theorem.

Theorem 3. The identities (26), (30) and $g_{i j}=g_{j i}$ constitute a complete set of identities for the components $g_{i j}, B_{i j k l}$, and $B_{i j k l, m, p}$.

The components $g_{i j}$ have to be included since they appear explicitly in the right member of (27).

Princeton University 